\title{
Optically Controlled Silicon MESFET Fabrication and Characterizations for Optical Modulator/Demodulator
}

\author{
S.N. Chattopadhyay, C. B. Overton, S. Vetter, M. Azadeh, B. H. Olson, and N. El Naga
}

\begin{abstract}
An optically controlled silicon MESFET (OPFET) was fabricated by diffusion process to enhance the quantum efficiency, which is the most important optoelectronic device performance usually affected by ion implantation process due to large number of process induced defects. The desired impurity distribution profile and the junction depth were obtained solely with diffusion, and etching processes monitored by atomic force microscope, spreading resistance profiling and $\mathrm{C}-\mathrm{V}$ measurements. With this approach fabrication induced defects are reduced, leading to significantly improved performance. The fabricated OPFET devices showed proper I-V characteristics with desired pinch-off voltage and threshold voltage for normally-on devices. The peak photoresponsivity was obtained at $620 \mathrm{~nm}$ wavelength and the extracted external quantum efficiency from the photoresponse plot was found to be approximately $87.9 \%$. This result is evidence of enhancement of device quantum efficiency fabricated by the diffusion process. It also supports the fact that the diffusion process is an extremely suitable process for fabrication of high performance optoelectronic devices. The maximum gain of OPFET at optical modulated signal was obtained at the frequency of $1 \mathrm{MHz}$ with rise time and fall time approximately of $480 \mathrm{nS}$. The extracted transconductance shows the possible potential of device speed performance improvements for shorter gate length. The results support the use of a diffusion process for fabrication of high performance optoelectronic devices.
\end{abstract}

\footnotetext{
Manuscript received Nov. 1, 2009; revised Sep. 24, 2010. Department of Electrical and Computer Engineering California State University Northridge 18111 Nordhoff Street Northridge, CA 913308346

E-mail: somnath.chattopadhyay@csun.edu
}

Index Terms - OPFET, MESFET, etching, metallization, diffusion process, quantum efficiency, spectral responsivity, gain-bandwidth, device fabrication, characterization

\section{INTRODUCTION}

Optically controlled MESFETs (OPFETs) have drawn considerable attention in recent years due to their potential application as optically controlled microwave devices [1] and optical modulator/demodulators [2]. A number of theoretical and experimental works have been reported on optically controlled MESFETs [3-6]. The potential of MESFETs as high-speed photodetectors was first demonstrated by Baack et al [7]. Various control functions such as amplifier gain, phase shifting, oscillator tuning and injection locking can be achieved by using OPFET devices. Experimental findings show that the microwave characteristics of a GaAs MESFET can be controlled by incident light radiation having photon energy greater than or equal to the bandgap energy of GaAs in the same manner as varying the gate bias [8-12]. In comparison with avalanche photo diodes (APDs), OPFET devices show superior performance for dispersion measurement on optical fibers with low dispersion and very high speed [13]. For frequencies higher than a few $\mathrm{GHz}$, the best photodetector devices are p-i-n or Schottky diodes or ITO photodiodes combined with MESFET devices used as a preamplifier in built-in monolithic optoelectronics integrated circuit (OEIC). The gain and noise performance of single-gate GaAs MESFET's have been extensively characterized and the MESFET potential in low noise amplifiers has been clearly demonstrated [14]. The advantage of MESFET using a preamplifier is low noise performance and extremely low gate-to-source capacitance. This low capacitance allows for high sensitivity. In recent years, there 
has been considerable attention given to the development of silicon based OEICs $[15,16]$, which is the integration of a photodetector (p-i-n or APD or MSM) with a transistor (MESFET or HBT). The fabricated OPFET device shows similar function of OEICs for photo detection and amplification with reduced signal delay, noise and enhancement of QE and responsivity, etc. Successful implementation of OPFET in MMIC applications, however, largely depends on the noise performance of the device in the illuminated condition. The semi-insulated substrate of GaAs MESFET technology greatly reduces parasitic capacitances and thus, is an excellent choice for low noise and high speed applications [17]. For a given gate length technology, increasing gate width of MESFET device has been found effective at improving the noise figure [18].

To date, desired impurity distribution profiles have been achieved with ion implantation followed by annealing to minimize defects. Experiments measuring the bulk generation lifetime of MOS capacitors have shown residual ion implantation defects in the substrates remain in spite of annealing [19]. Phosphorus ion implantation not only results in the transition of the crystalline fullerenes to an amorphous material phase, but also produces a significant defect level, which is evident from solar cell efficiency ranges of only $10-20 \%$ [20]. An effective loss of photo-generated carriers due to the ion implantation process induced defects in the active channel region of OPFET is a major issue of degradation of quantum efficiency and sensitivity [21]. A dc analytical model of an ion implanted GaAs based OPFET has been proposed by many researchers [22-24]. Unfortunately, none of these models incorporate a number of important parameters required to account for physical fabrication problems such as out-diffusion of carriers during annealing and ion implanted process induced defects. These additional parameters are necessary to properly estimate quantum efficiency performance in practical devices. Process induced defects due to diffusion process are obviously far less compared to ion implantation, as has been verified by our previous articles $[25,26]$. This work focuses on investigating of the impact of diffusion process induced defects verifying their minimal effect on quantum efficiency. Various parameters were optimized for our fabricated device to maximize speed and reduce power consumption. The gate length of the MESFET was shortened and parasitics reduced to increase $f_{T}$, and the load resistance was selected to minimize the output-circuit time constant without reducing the logic swing. This research paper presents the characterization results of our fabricated OPFET in our microelectronics laboratory.

\section{THEORY}

A schematic diagram of OPFET with MESFET structure is shown in Fig. 1. The transparent gate is made of indium tin oxide (ITO) forming a Schottky rectifying contact with proper antireflection properties. OPFET device structure was optimized by analytical modeling

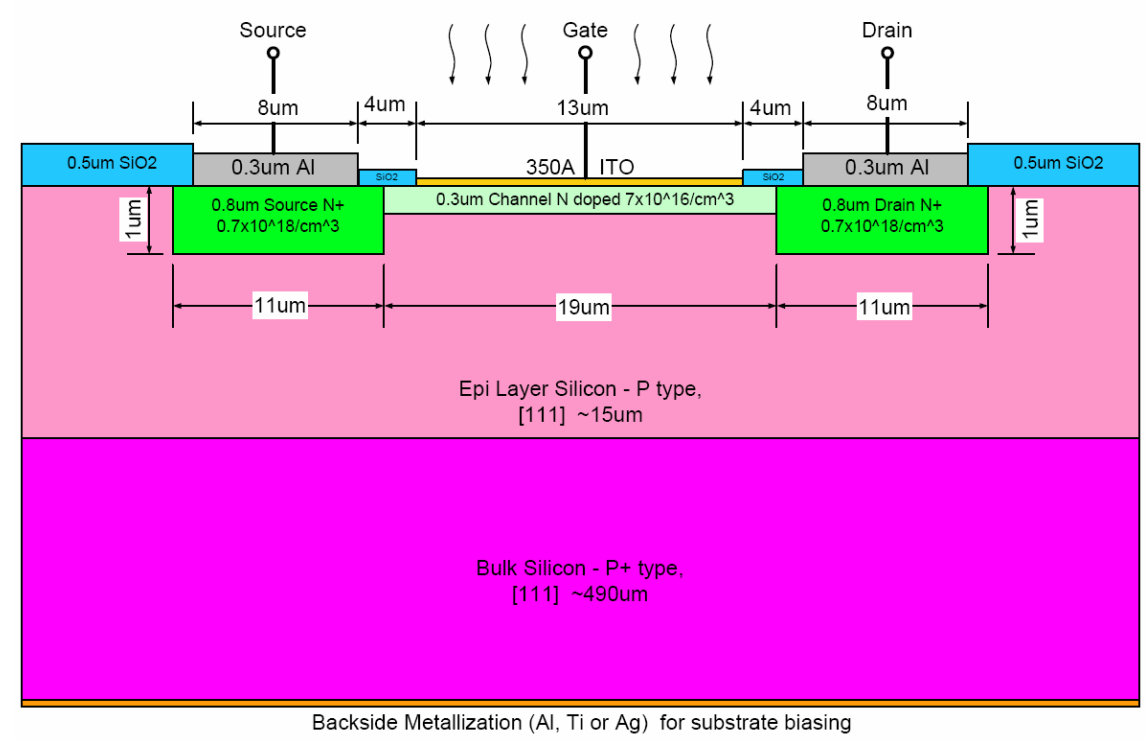

Fig. 1. Device structure of OPFET. 
and TCAD simulations for gate lengths varying from 1 $\mu \mathrm{m}$ to $13 \mu \mathrm{m}$. Fig. 1 shows a cross-sectional schematic diagram for $13 \mu \mathrm{m}$ gate length. The theory for OPFET considering non-uniform doping has been described with the physics based analytical modeling [21]. When the device is illuminated by photons with energy $E_{p h}=h v(h$ $=$ Planck's constant and $v=$ frequency of the light) greater than the energy bandgap of silicon $(1.12 \mathrm{eV})$ but smaller than the energy bandgap of indium tin oxide $(\sim 3.5 \mathrm{eV})$, electron and hole pairs are generated.

Depending on device structure and the wavelength of the incident light, several different photo-induced processes may occur. The primary photo effect along the Schottky junction (low injection case of incident photon flux) is photovoltaic. In this case, the photo-generated electron-hole pairs are separated by the Schottky junction built-in electric field and move in different directions due to the space charge effect [27]. The electrons move towards the channel and holes toward the surface. The holes recombine with traps or recombination centers, giving rise to a gate leakage current $[13,24]$. Thus a photovoltage is developed across the junction, effectively reducing the depletion width in the channel. This reduction of the gate depletion region causes an increase in drain current for a given source-drain bias. Thus a photovoltage is developed across the junction, effectively reducing the depletion width in the channel. This reduction of the gate depletion causes an increase in drain current. Thus variation of depletion width directly affects the drain current. This optical modulation of drain current gives higher optical responsivity in a FET compared to a simple Schottky diode, which normally achieves an optical responsivity of less than unity. Additional lesser photo-induced effects may be present as well. For large gate resistances a noticeable photovoltage is generated, supplementing the dark gate-source bias voltage. A similar photovoltaic effect can also occur at the channel substrate junction which can act as a photodiode but this effect can usually be ignored by assuming low injection conditions.

Moreover, the photo-generated carriers in the depletion layer from the gate to the drain are swept out by the electric field and contribute to the primary photocurrent between the drain and gate. This gate current causes a change in the gate voltage due to the potential drop on the gate resistance, which in turn causes a change in the drain current via the transconductance of MESFET [28].

Optical radiation is absorbed by the gate depletion region through the transparent ITO gate. As a result, electron-hole pairs (EHPs) are generated and carrier transport occurs due to drift and surface recombination. Due to gate-biasing, the generated electrons move towards the n-channel, whereas the holes move towards the surface to recombine the surface traps. Carrier transport is considered to be due to diffusion, and both bulk and surface recombination, therefore reasonable channel thickness is optimized to absorb maximum optical radiation as well as to prevent the loss of photo-generated carriers. Space charge in the gate and substrate depletion region changes due to the excess generated carriers, which in turn controls the channel opening leading to incremental changes in current. Changes in the space charge region modulate the barrier potential in the junction and as a result, photovoltage will be generated affecting the electrical parameters in the dark to illumination transitions.

\section{DEVICE FABRICATION}

The active area was formed by diffusion process, which is preferred over ion implantation due to diffusion having little or no process induced defects [21]. Ion implantation induced defects in silicon substrates have been characterized to study the dependence of substrate dopant species (phosphorous and boron) on defect formations [29]. I-V characteristics of ion implanted solar cell devices at optical illumination have shown efficiencies as low as $0.01 \%$. Phosphorous ion implantation not only results in the transition of the crystalline fullerenes to amorphous material phase, but also produces a significant defect level [30]. An effective loss of photogenerated carriers due to ion implantation process induced defects in the active channel region of OPFET is a major issue of degradation of quantum efficiency, sensitivity, etc.

OPFET fabrication was performed using silicon wafers with $\langle 100\rangle$ orientation with a $10-12 \mu \mathrm{m}$ thick pepitaxy with a resistivity of $10 \mathrm{Ohm}-\mathrm{cm}$ and $\mathrm{p}+$ substrate with thickness of about 490 to $500 \mu \mathrm{m}$ and resistivity of $0.02 \mathrm{Ohm}-\mathrm{cm}$ boron doped. The wafer was cleaned by SC-1 and SC-2 processes followed by phosphorus impurity doping by diffusion process using Saint Gobain (Carborundum) planar diffusion sources (PDS) PH 900 
to form the active channel. The resultant diffusion process shows an impurity distribution with the surface impurity concentration of approximately $3 \times 10^{18} \mathrm{~cm}^{-3}$ and junction depth of approximately $0.375 \mu \mathrm{m}$ measured by Solecon Laboratories shown in Fig. 2.

The high surface impurity concentration and large junction depth resulting from this type of doping process are not reasonable for optoelectronic device for high QE and speed performance. Hence, the doped silicon wafers were further etched by $45 \% \mathrm{KOH}$ at $50{ }^{\circ} \mathrm{C}$ for $3.5 \mathrm{~min}$ utes at $\mathrm{N}_{2}$ ambient based on the optimization of etching time and temperature calculated using the $\mathrm{KOH}$ etching model [31], thus a shallower junction with lower surface doping concentration was achieved compared to the conventional diffusion process using PH900.

$\mathrm{KOH}$ etching process was preferred for anisotropic etching and uniform bright surface [32]. One of the crucial issues during etching process is its effect on the surface roughness of the resulting silicon film surface. The roughness of silicon $<100>$ surfaces caused by etching can affect their nanotribological (friction and wear) behavior $[33,34]$ as well as mechanical properties of the

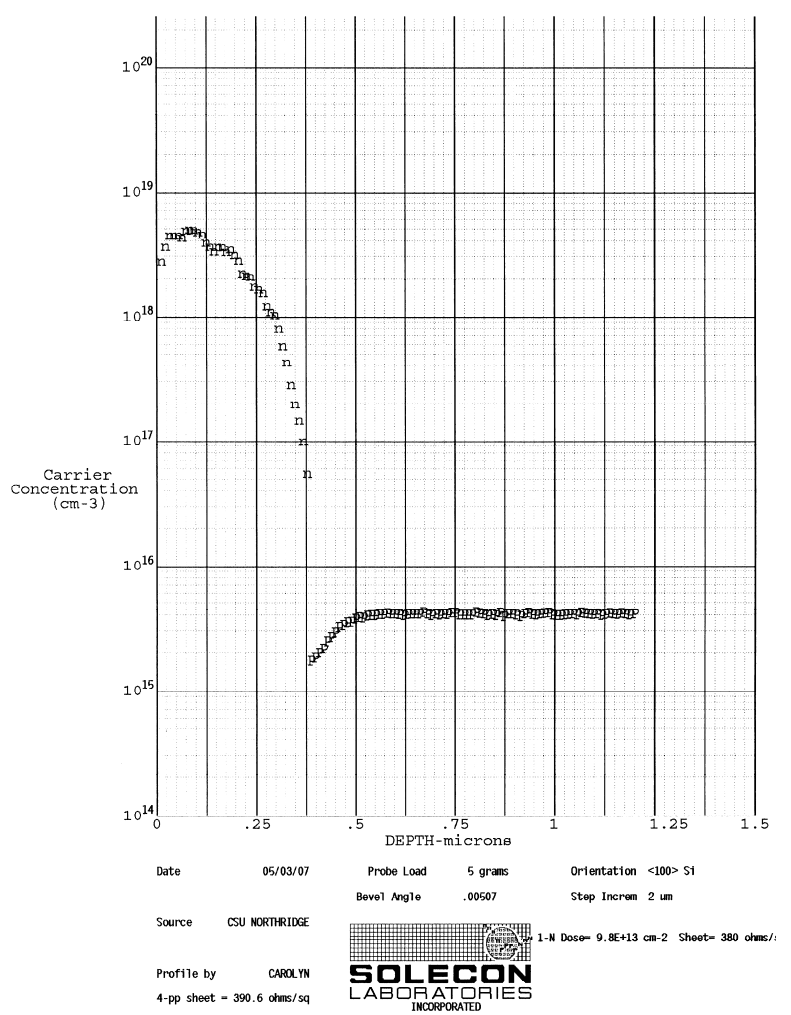

Fig. 2. Doped Phosphorous Impurity distribution measured by Solecon Laboratories. microscale structures [35-37]. Experimental studies on the measurement of the roughness of the etched surfaces $[38,39]$ have shown that the roughness of the etched surface depends upon the temperature and concentration of $\mathrm{KOH}$ etchant [40-42]. Higher micro-roughness will result in voids that can not be cured during annealing processes [43] and cause higher stress in the bonding interface [44]. Once the surface micro-roughness exceeds a critical value, the wafer will no longer be bondable. However, anisotropic etchant solutions such as $\mathrm{KOH}$ or $\mathrm{NaOH}$ generally form random distributed pyramids as a micro-roughness, which can improve light trapping properties $[45,46]$. These pyramidal textures have geometries, which allow as much light as possible to be absorbed and converted to electrical current in the solar cell or optoelectronic devices. Fig. 3 shows KOH etching analysis of a doped sample inspected by Atomic Force Microscopic (AFM). Considering above effects, some test wafers were etched and finally the etch process was optimized to an etching solution of $45 \% \mathrm{KOH}$ at $50{ }^{\circ} \mathrm{C}$ for 3.5 minutes at $\mathrm{N}_{2}$ ambient. This technique has been applied to OPFET forming a channel with appropriate impurity distribution and junction depth. The etched and non-etched areas can be clearly seen in Fig. 3. Fig. 4 shows the impurity distribution of $\mathrm{KOH}$ etched sample extracted from $\mathrm{C}-\mathrm{V}$ measurement, where the $\mathrm{KOH}$ etching brings the surface concentration down from about $2 \times 10^{19} \mathrm{~cm}^{-3}$ to the range of $6 \times 10^{16}-7 \times 10^{16} \mathrm{~cm}^{-3}$ and the junction depth in the range of about $0.1 \mu \mathrm{m}$.

The source and drain doping was formed by diffusion process using PH900 and the drain/source doping con-

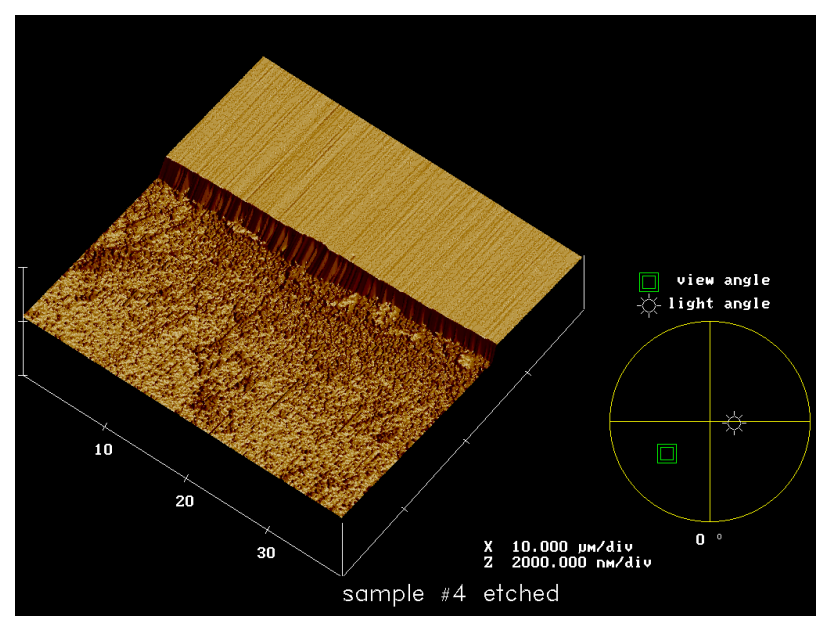

Fig. 3. AFM image of silicon wafer sample etched by $\mathrm{KOH}$ at $50{ }^{\circ} \mathrm{C}$ for $3.5 \mathrm{~min}$ in $\mathrm{N}_{2}$ ambient. 

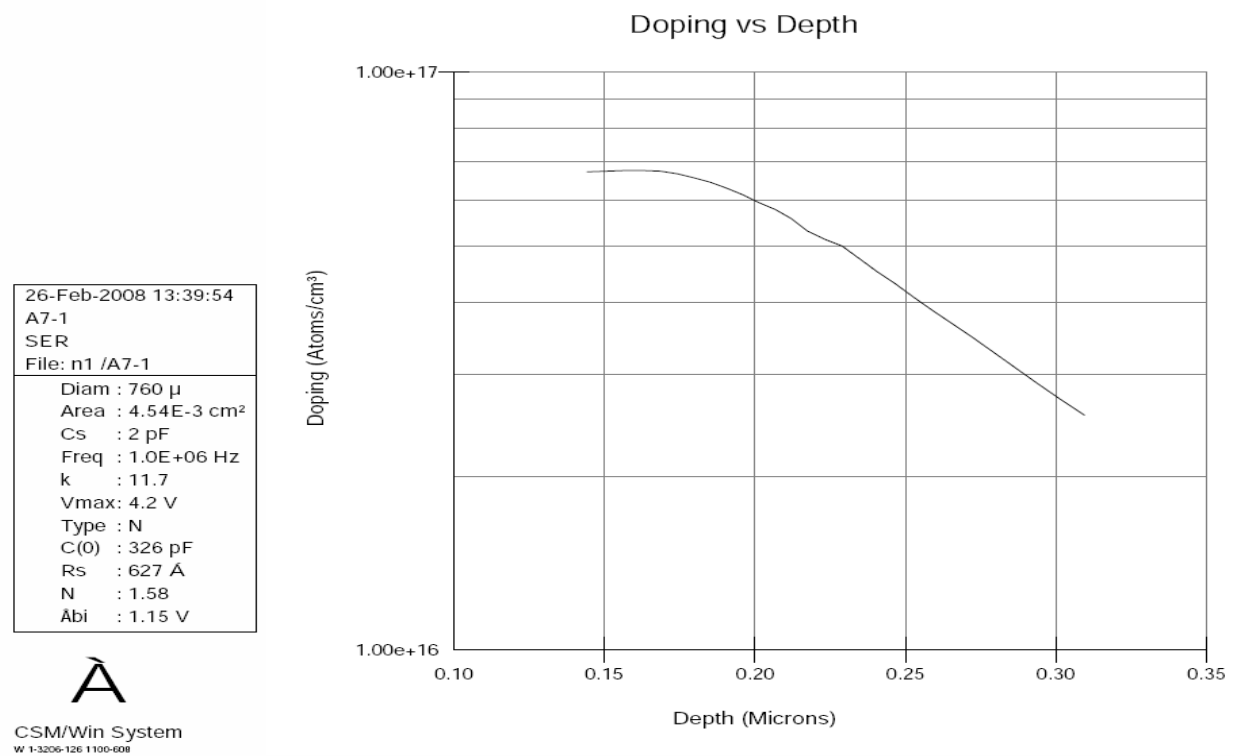

Fig. 4. Impurity distributions from $\mathrm{C}-\mathrm{V}$ measurement after $\mathrm{KOH}$ etching.

centration and junction depth were kept in the range of $5 \times 10^{19}-8 \times 10^{19} \mathrm{~cm}^{-3}$ and 0.5 to $0.75 \mu \mathrm{m}$. Aluminum film deposition for source/drain was performed by a sputtering system with power set to 100 Watt and 10SCCM argon flow resulting in the chamber pressure of approximately $4.3 \times 10^{-3}$ Torr for 30 minutes. The deposited aluminum film thickness measured by Veeco A-Scope was found to be $3000 \AA$ with a thickness tolerance of 5\%.

The indium tin oxide (ITO) Schottky gate was formed by using a bi-layer photoresist metal lift-off process. ITO deposition was performed by sputtering with initial vacuum in the range of $5 \times 10^{-8}$ to $7 \times 10^{-8}$ Torr. The ITO film was deposited at power setting of 100 Watt for 4 minutes in argon and oxygen flowing at 12 SCCM and 0.2 SCCM respectively, resulting in a chamber pressure of approximately $8.5 \times 10^{-3}$ Torr. An ITO film thickness of $600-700$ $\AA$ was typically used for defining the gate during OPFET fabrication. The entire process cycle was optimized for process integration and resulted in approximately $90 \%$ yield for nine various OPFET devices with gate lengths ranging from $1 \mu \mathrm{m}$ to $13 \mu \mathrm{m}$.

\section{RESULTS AND DISCUSSION}

The electrical and optical characteristics of the fabricated OPFET were extracted from different experiments to evaluate the device performance, which is described below. The I-V characteristics of the $13 \mu \mathrm{m}$ OPFET device were obtained by probing the source, drain and gate contact pads with floating ground as measured by a Tektronix 370B curve tracer as shown in Fig. 5(a) and 5(b). As indicated, curves were captured at gate-source voltages $\left(\mathrm{V}_{\mathrm{GS}}\right)$ of $2 \mathrm{~V}$ to $12 \mathrm{~V}$ in $2 \mathrm{~V}$ steps. The obtained plots are shown under dark and illuminated conditions. The OPFETs with $13 \mu \mathrm{m}$ gate length and gate width of $200 \mu \mathrm{m}$ had maximum saturation currents of $39 \mathrm{~mA}$ and $27 \mathrm{~mA}$ for gate-to-source voltages $\mathrm{V}_{\mathrm{GS}}=12 \mathrm{~V}$ and $2 \mathrm{~V}$ respectively. Comparison of Fig. 5(a) and 5(b) shows the experimental drain current (y-axis) versus drain-source voltage (x-axis) under dark and illumination conditions, indicating the devices have typical FET characteristics. Due to scaling, where the photo-generated current is very small relative to the bias current, the photocurrent measurement was performed using the two diodes configuration $($ Schottky $/ \mathrm{n} / \mathrm{p})$ between the gate and substrate shown in Fig. 6(a) and 6(b). The measured I-V characteristics between the gate and substrate under forward and reverse bias in dark and illumination conditions exhibit the typical photo current similar to that of an ideal photodiode in transition from dark to illumination. The measured reverse saturation current under illumination was approximately $80-90 \mu \mathrm{A}$ due to the photo generated carriers resulting from white light. 


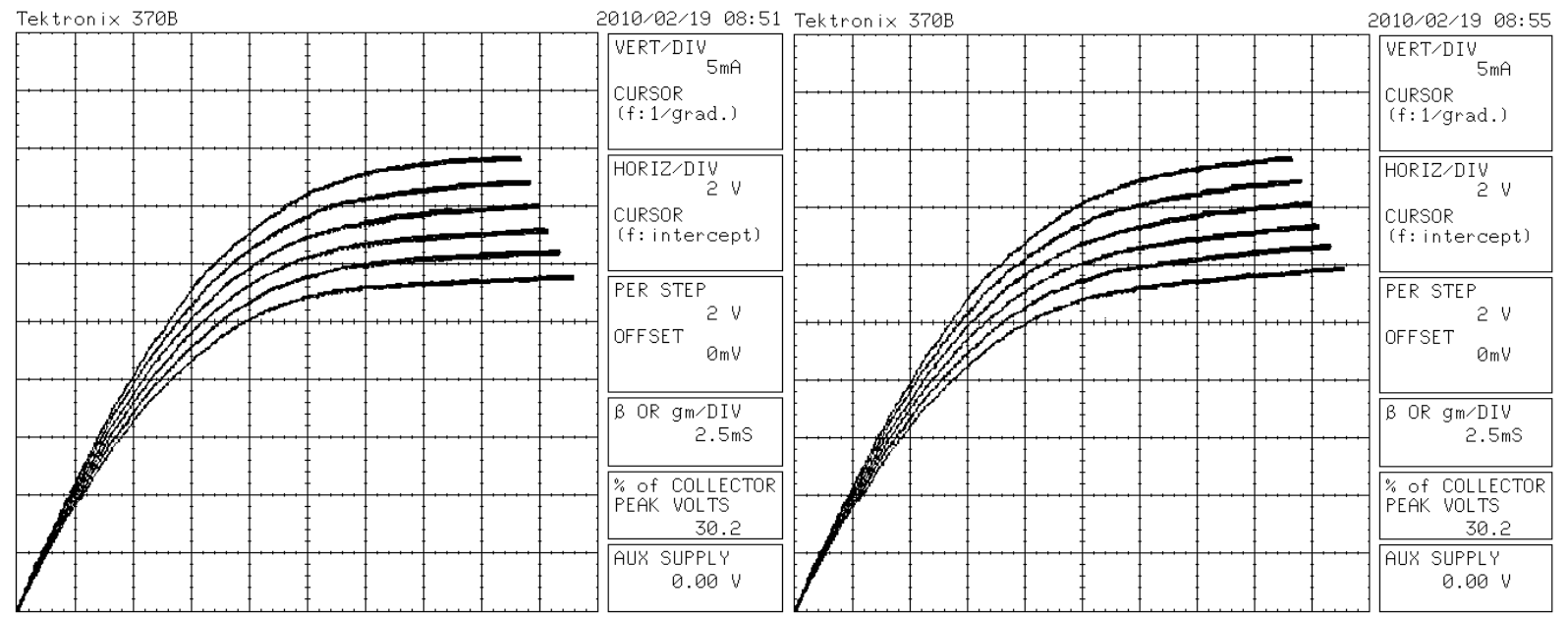

Fig. 5(a) and 5(b). $13 \mu \mathrm{m}$ OPFET - IV curves taken from first complete OPFET wafer. From Left to right: $13 \mu \mathrm{m}$ gate OPFET IV curves without illumination, and IV curves with illumination. $\mathrm{X}$ axis: Drain to Source Voltage $\left(\mathrm{V}_{\mathrm{DS}}\right)$ and $\mathrm{Y}$ axis: Drain Current $\left(\mathrm{I}_{\mathrm{D}}\right)$.
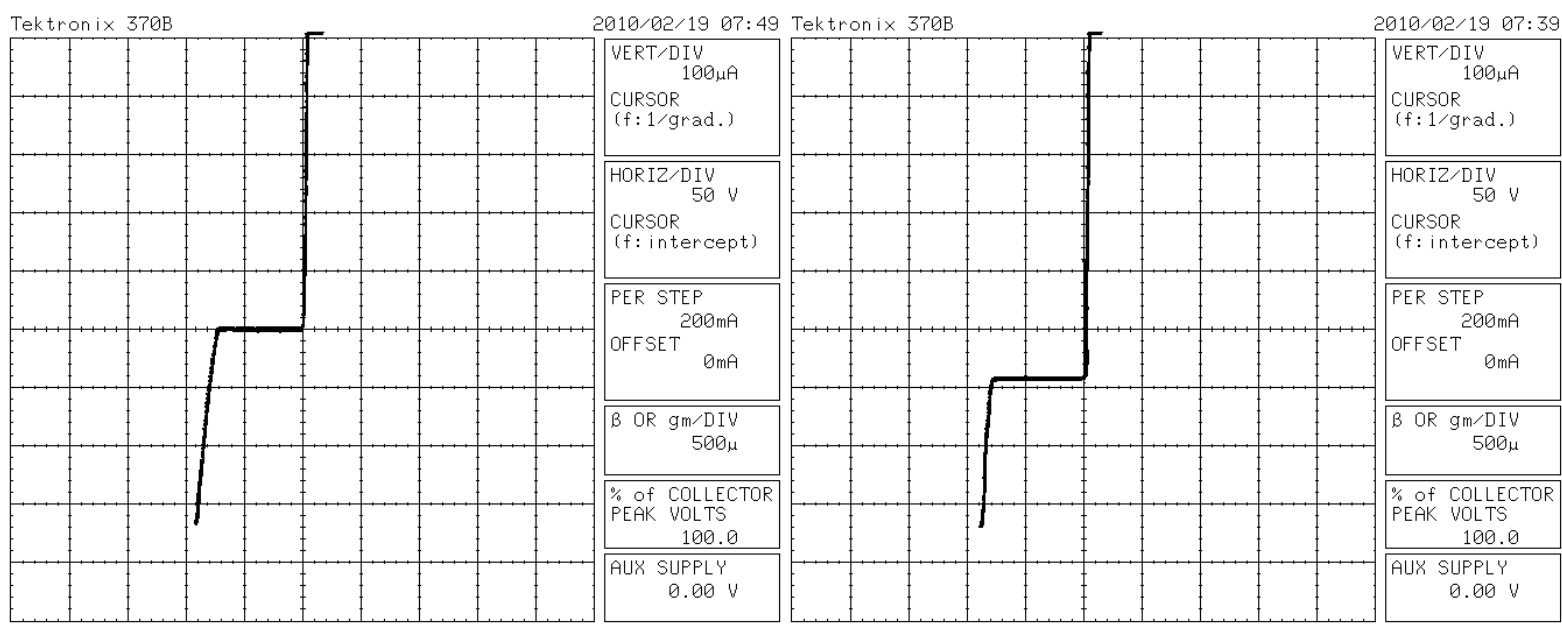

Fig. 6(a) and 6(b). Schottky Photo Diode effect - Schottky diode IV curve is shown using the $13 \mu \mathrm{m}$ OPFET gate and the substrate. As can be clearly seen, the reverse bias current changes significantly between darkened condition (left) and illuminated (right). X axis: Gate to Substrate Voltage and Y axis: Gate Current.

The pinch-off voltage is found to be approximately $6.06 \mathrm{~V}$ extracted from the I-V characteristics. The resulting pinch-off voltage justifies the channel depth as optimized by process simulation and the diffusion process. The active channel depth is an important parameter, which is key in determining the pinch-off voltage of the physical device. The active channel depth and the junction depth of the doped layers are co-related to estimate the pinch-off voltage. Therefore, the pinch-off voltage is not only one of the electrical parameters of the OPFET, but also is key to assist in the optimizing of the fabrication parameters such as the channel depth and junction depth. Diffusion process parameters such as the diffusion time of 20 minutes, diffusion coefficient of approximately $5 \times 10^{-15} \mathrm{~cm}^{2} / \mathrm{sec}$ for $840{ }^{\circ} \mathrm{C}$ [47] and measured impurity flux of $5.5 \times 10^{13} \mathrm{~cm}^{-2}$ from the spreading resistance profiling (SRP) for impurity distribution profile were taken into consideration to calculate the pinch-off voltage from our previous paper [21].

Fig. 7 shows the plot of OPFET device's responsivity, factoring out the Air-ITO boundary reflectance and ITO absorption (approximately only 14\% combined loss at $620 \mathrm{~nm}$ due to ITO thickness of only 60 to $70 \mathrm{~nm}$ and index of refraction of 1.95), where the peak responsivity was found at $620 \mathrm{~nm}$ and the spectral response was observed in the range of $500-910 \mathrm{~nm}$. The spectral response was measured under reverse bias of $10 \mathrm{~V}$ with optical flux from $350 \mathrm{~nm}$ to $1100 \mathrm{~nm}$. The resulting spectral responsivity profile is somewhat typical for silicon material, given the small effect of ITO spectral absorp- 


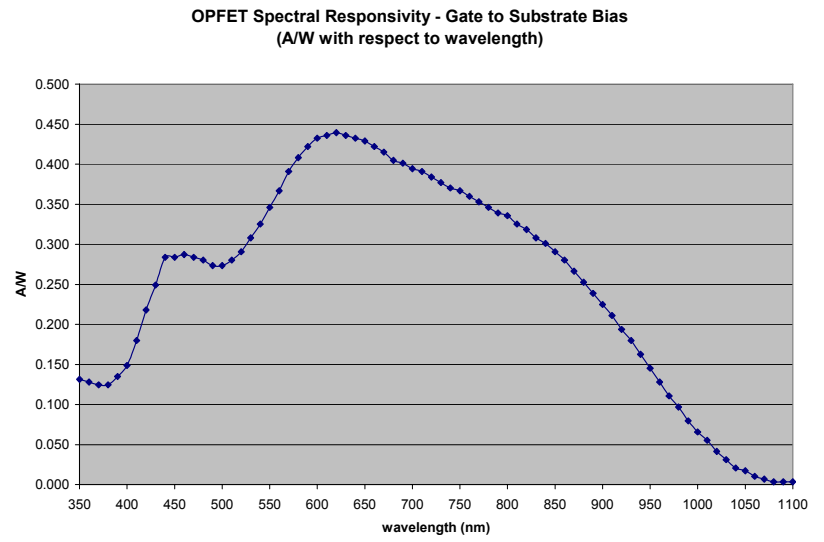

Fig. 7. OPFET optical response (measured by the reverse biased current/watt) versus optical wavelength.

tion and effect of the doping on absorption profile. The optical transmissivity of doped silicon is a function of the sheet resistance and incident wavelengths [48]. Hence, the peak responsivity of the OPFET device can be controlled by adjustment of doping in the absorption layer.

Using this data, quantum efficiency at peak responsivity can be calculated using following equation:

$$
Q E_{\lambda}=\frac{R_{\lambda}}{\lambda} \times \frac{h c}{e} \approx \frac{R_{\lambda}}{\lambda} \times(1240 \mathrm{~W} \cdot \mathrm{nm} / \mathrm{A})
$$

where, $\frac{R_{\lambda}}{\lambda}=\frac{0.439 \mathrm{~W} / \mathrm{A}}{620 \mathrm{~nm}}$ which yields a quantum efficiency of about 0.879 or $87.9 \%$.

The spectral responsivity results show that the OPFET has an external quantum efficiency (excluding the AirITO boundary reflectance and ITO absorption) of approximately $87.9 \%$, which is much higher than other optoelectronic devices. Factoring in the losses at the Air-
ITO boundary and ITO absorption, total external quantum efficiency is calculated as being about $75.6 \%$. This result indicates an improvement due to fewer process induced defects in the active channel region of OPFET compared to optoelectronic devices fabricated by other more complex and expensive doping process technologies, such as ion implantation.

Fig. 8 shows the experimental set-up to measure the modulated output voltage of the device with $13 \mu \mathrm{m}$ gate versus frequency. Fig. 9(a) shows the OPFET device was packaged in a TO-5 header with Fig. 9(b) showing a close-up of the $13 \mu \mathrm{m}$ device. Connections were made by gold wire bonding using cold welding process at OSI Ins., USA. EPO-TEK-H20E silver epoxy was used to bond to the gate pad, due to the inability of gold wire to adhere to the ITO coated gate pads. The transistor was mounted in a TO-5 to SMA test fixture specially designed for high frequency compatibility and high precision cables were used to minimize any high frequency effects due to interconnects.

The drain/source and gate terminals of the OPFET device were biased through DC power supplies. The modulated laser signal impinged on the gate and resulted in common source output signal modulation. Fig. 10 shows the output voltage with the drain-to-load resistor of 1.5 $\mathrm{k} \Omega$ as measured by a $500 \mathrm{MHz}$ ( $5 \mathrm{GS} / \mathrm{s}$ ) bandwidth oscilloscope, with a modulated light signal swept from 100 $\mathrm{kHz}$ to $2.5 \mathrm{MHz}$, produced by the direct modulation of a $650 \mathrm{~nm}, 5 \mathrm{~mW}$ laser diode illuminating the ITO transparent gate.

The OPFET device was biased with $\mathrm{V}_{\mathrm{DS}}=24 \mathrm{~V}$ and $\mathrm{V}_{\mathrm{GS}}=-1.35 \mathrm{~V}$. The measured voltage gain was $\mathrm{A}_{\mathrm{V}}=2.2$ as obtained in the dark condition. The laser was biased at

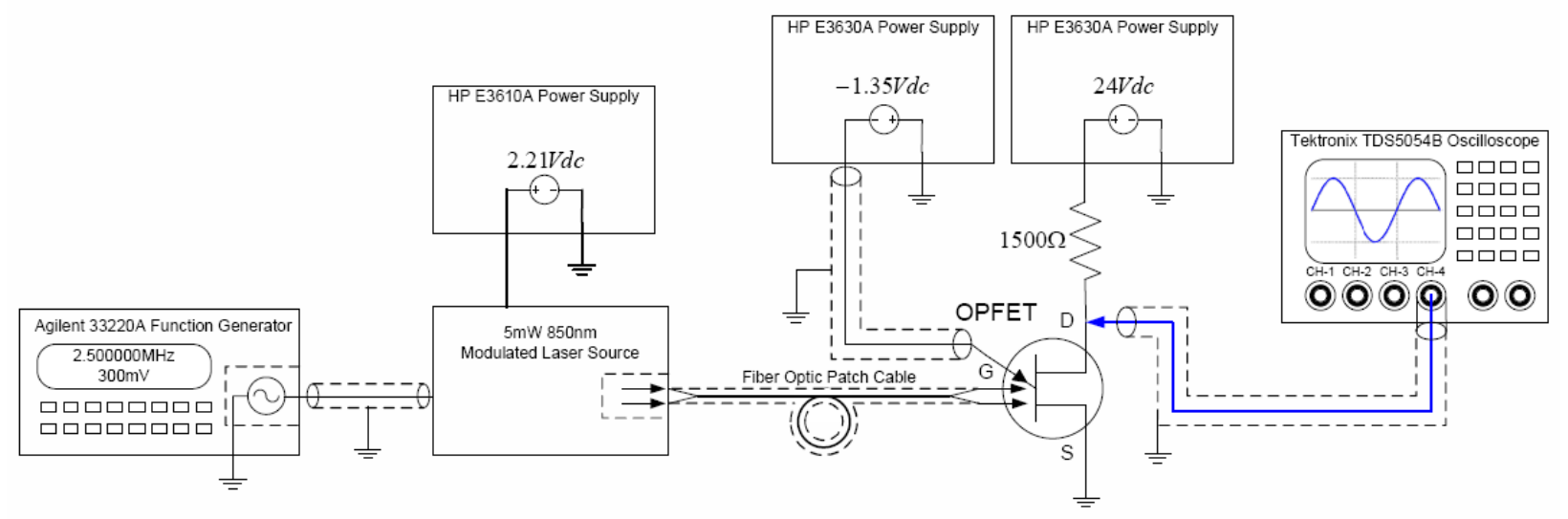

Fig. 8. Experimental setup for optical characterization of the OPFET. 

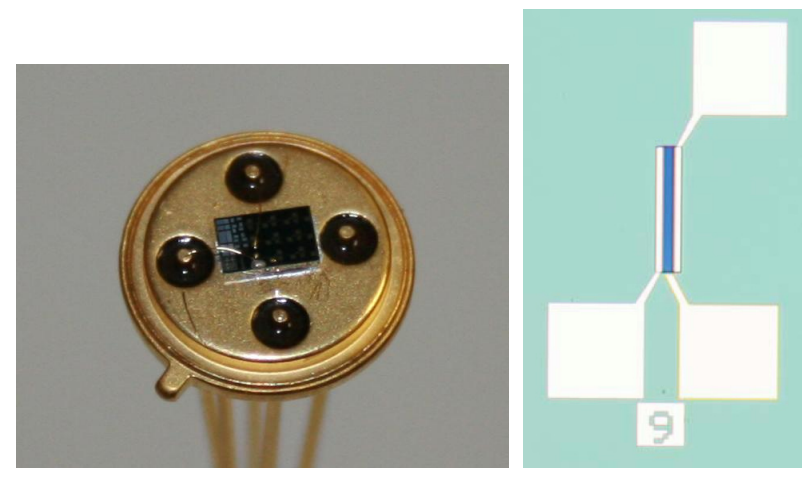

Fig. 9(a) and 9(b). OPFET die in TO-5 package, and close up of $13 \mu \mathrm{m}$ device.

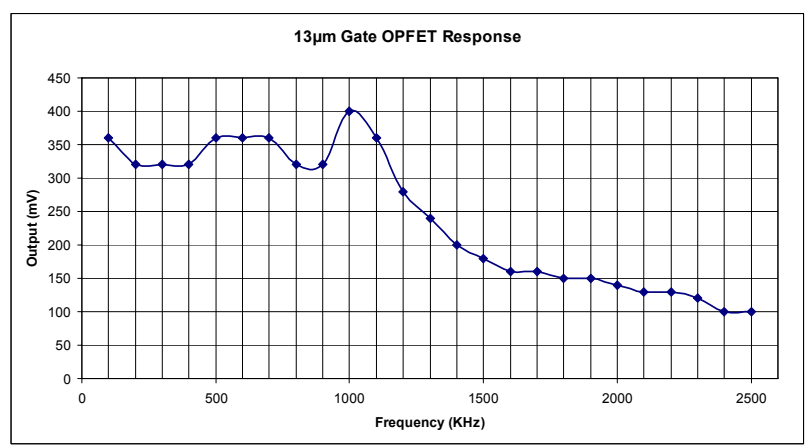

Fig. 10. OPFET output voltage versus modulated signal frequency with a laser biasing of $20 \mathrm{~mA}$ and $2.21 \mathrm{~V}$, laser signal amplitude $300 \mathrm{mV}, \mathrm{V}_{\mathrm{DD}}=24 \mathrm{~V} \mathrm{DC}, \mathrm{V}_{\mathrm{GS}}=-1.35 \mathrm{~V}, \mathrm{~A}_{\mathrm{V}}=2.2$ (electrical gain at $1 \mathrm{MHz}$ at dark condition).

$2.21 \mathrm{~V}$ with a bias current of $20 \mathrm{~mA}$. The amplitude of the signal generator output was $300 \mathrm{mV}$ for the various frequencies modulating the laser illumination. The voltage gain of the device under illumination is found to be 1.33 at approximately $1.0 \mathrm{MHz}$ and starts falling above $1.1 \mathrm{MHz}$. The voltage gain and current gain variations in the dark and illumination conditions shows a practical evidence of the OPFET as an optical modulator. The 3 $\mathrm{dB}$ bandwidth of the OPFET device was determined to be approximately $1.5 \mathrm{MHz}$, which is limited by the cutoff frequency of the $13 \mu \mathrm{m}$ Si MESFET, which should be in the GHz range for similar sub-micron devices.

Fig. 11 shows a plot of drain-source current versus gate-source voltage for $1 \mu \mathrm{m}$ and $13 \mu \mathrm{m}$ gate length OPFET devices estimated from the I-V characteristics measured from the Tektronix 370B curve tracer. The intersecting point of the plot at $\mathrm{V}_{\mathrm{GS}}$ axis with $\mathrm{I}_{\mathrm{DS}}=0$ in Fig. 11 indicates the threshold voltages of -4.0 volts for 13 $\mu \mathrm{m}$ and -2.0 volts for $1 \mu \mathrm{m}$ gate length under gate illumination condition. The maximum drain current for 13 $\mu \mathrm{m}$ gate OPFET device becomes almost twice as small

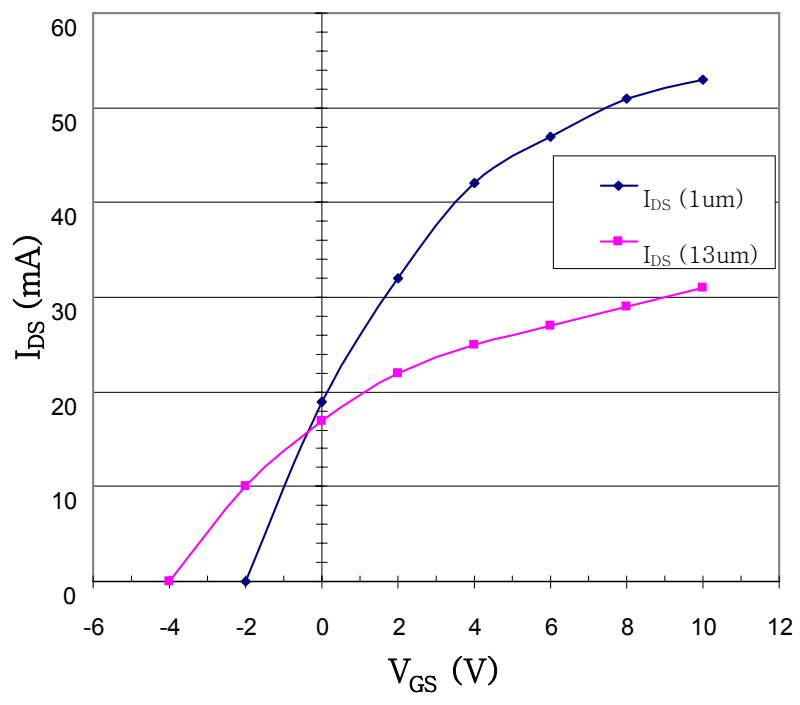

Fig. 11. Drain-source current versus gate-source voltage for 1 $\mu \mathrm{m}$ and $13 \mu \mathrm{m}$ gate length.

i.e. approximately $32 \mathrm{~mA}$ compared to that of $1 \mu \mathrm{m}$ gate OPFET i.e. approximately $54 \mathrm{~mA}$ due to the gate length reduction. As a result, the transconductance is higher and the gate capacitance should be lower, compared to those of the $13 \mu \mathrm{m}$ gate OPFET. Therefore, higher gate length and gate-source capacitance will limit the device response to a range in the lower $\mathrm{MHz}$ scale because of the influence of gate capacitance and long transit time on the frequency response.

\section{Conclusions}

In contrast to optoelectronic devices fabrication by ion implantation and their inherent device performance issues, OPFET fabrication was pursued using diffusion process. Due to low process induced defects and low gate-to-source (Schottky gate) capacitance, high quantum efficiency has been achieved by obtaining high photocurrent with excellent responsivity at wavelengths ranging from $550 \mathrm{~nm}$ to $850 \mathrm{~nm}$. The enhancement of quantum efficiency (87.9\%) allows for the sensitive control of the channel opening by significant photovoltaic effect in the gate. The peak responsivity and maximum output voltage of the $13 \mu \mathrm{m}$ gate OPFET were found to be at $620 \mathrm{~nm}$ and $1.0 \mathrm{MHz}$ respectively. Modeling and simulation shows that the responsivity and output voltage can be further enhanced by optimizing the gate length 
and channel doping concentration. Comparative study of transconductance of OPFET devices with $1 \mu \mathrm{m}$ and 13 $\mu \mathrm{m}$ gate lengths shows that the transconductance of $1 \mu \mathrm{m}$ gate length is much higher compared to that of the $13 \mu \mathrm{m}$ gate length. Moreover, the gate-source capacitance for the $1 \mu \mathrm{m}$ gate length is much smaller than that of the 13 $\mu \mathrm{m}$ gate. It can be anticipated that the combination effect of transconductance and gate-source capacitance of the $1 \mu \mathrm{m}$ gate OPFET would show significantly improved frequency performance useful for high speed switching applications. The scope of research for measuring the responsivity and frequency response characteristics of OPFETs with $1 \mu \mathrm{m}$ gate length (or smaller) would be extremely valuable to completely understand the full potential of silicon OPFET devices in optical switching, optical routing, optical modulation and high sensitivity photo-detection applications.

\section{ACKNOWLEDGMENTS}

This research is primarily supported by Army Research Office, US Department of Defense, under award No. W911NF-05-1-0025. The authors wish to thank Dr. M. Gerhold, for his continuous assistance and encouragement. The authors wish to also thank Mr. M. Mansouri, President, OSI Inc. of Hawthorne, CA, USA for his great help in providing device packaging and characterization assistance.

\section{REFERENCES}

[1] R. N. Simons and K.B. Bhasin, "Analysis Optically Controlled Microwave /Millimeter-Wave Device Structures," IEEE Trans. on Microwave Theory and Techniques, Vol. MTT-34, p. 1349, 1986.

[2] J.M. Osterwalder and B.J. Rickett, "GaAs MESFET demodulates gigabit rates from GaAlAs injection laser," Proceeding of the IEEE, Vol. 67, p. 966, 1979.

[3] P. Chakrabarti, et al, "Switching Characteristics of an Optically Controlled GaAs-MESFET," IEEE Trans. on Microwave Theory \& Tech., Vol. MTT-42, pp. 365-375, 1994.

[4] V.K. Singh, S.N. Chattopadhyay and B.B. Pal,
"Optically Controlled Characteristics in an IonImplanted Silicon MESFET," Solid State Electronics, Vol. 29, pp. 707-711, 1986.

[5] S. Mishra, V.K. Singh and B.B. Pal, "Effect of Radiation and Surface Recombination on the Characteristics of an Ion-Implanted -GaAs MESFET," IEEE Trans. Electron Devices, Vol. 37, pp.2-10, 1990.

[6] S.R. Saxena, R.B. Lohani, R.U. Khan and B.B. Pal, "Generalized dc model of GaAs optical field effect transistor considering ion implanted profile," Optical Engineering, Vol. 37, No. 4, p.1343, 1998.

[7] C. Baack et al., "GaAs M.E.S.F.E.T.: A High-Speed Optical Detector," Electronics Letters, Vol. 13, p.193, 1977.

[8] S. Bose, et al., "Cutoff frequency and optimum noise figure of GaAs optically controlled FET," Microwave and Optical Technology Letters, Vol. 26 , No. 5, p.279, 2000.

[9] R. Zulegg, "Radiation effects in GaAs FET devices," Proceedings of the IEEE 77 (1989), p.389.

[10] H. Mizuno, "Microwave characteristics of an optically controlled GaAs MESFET," IEEE Trans. on Microwave Theory \& Tech., Vol. MTT 31, p. 596, 1983.

[11] J. Graffeuil, et al, "Light induced effects in GaAs FETs," Electronics Letters. Vol. 15, p. 439, 1979.

[12] A.A.A. DeSalles, "Optical control of GaAs MESFET's," IEEE Trans. Microwave Theory \& Tech., Vol. MTT-31, p. 812, 1983.

[13] B.B. Pal and S.N. Chattopadhyay, "Time dependent analysis of an ion implanted GaAs OPFET," IEEE Trans. Electron Devices, Vol. 42, p. 491, 1994.

[14] C. A. Liechti, "Performance of Dual-gate GaAs MESFET's as Gain-Controlled Low Noise Amplifiers and High Speed Modulators," IEEE Trans. on Microwave Theory and Techniques, Vol. MTT-23, No. 6, pp. 461-469, 1975.

[15] G. Breglio, et al, "Two silicon optical modulators realizable with a fully compatible bipolar process," IEEE Journal of Selected Topics in Quantum Electronics, Vol. 4, p. 1003, 1998.

[16] C.L. Schow, et al., "A 1-Gb/s monolithically integrated silicon nMOS Optical Receiver," IEEE Journal of Selected Topics in Quantum Electronics, Vol. 4, p.1035, 1998.

[17] L. Forbes, et al, "A Model for the Channel Noise of 
MESFETs Including Hot Electron Effects," Microelectronics Reliability, Vol. 39, pp. 1773-1786, 1999.

[18] R.A. Minasian, "Optimum Design of a 4-Gbit/s GaAs MESFET Optical Preamplifier," Journal of Lightwave Tech., Vol. LT-5, No.3, pp.373-379, 1987.

[19] K. Kanemoto, et al., "Dependence of ion implantation: Induced defects in substrate doping," J. Appl. Phys., Vol. 89, pp.3156-3161, 2001.

[20] K.L. Narayanan and M. Yamaguchi, "Phosphorous ion implantation in $\mathrm{C} 60$ for the photovoltaic applications," J. Appl. Phys., Vol. 89, pp. 8331-8335, 2001.

[21] S. N. Chattopadhyay, et al, "Optically controlled Silicon MESFET Modeling Considering Diffusion Process," Journal of Semiconductor Technology and Science, Vol. 7, No. 3, pp. 196-208, 2007.

[22] S. Bose, et al, "Optical Radiation and Temperature Dependent Microwave Performance of Optically Biased GaAs Metal-Semiconductor Field Effect Transistors," Optical. Engineering. (SPIE), Vol. 41, No. 1, pp. 190-199, 2002

[23] P. Chakrabarti, et al, "An Analytical model of GaAs OPFET," Solid State Electronics, vol. 39, no. 10, pp. 1481-1490, 1996.

[24] P. Chakrabarti, et al, "An Improved Model of IonImplanted GaAs OPFET," IEEE Trans. on Electron Devices, Vol. 39, No. 9, pp.2050-2059, 1992.

[25] S.N. Chattopadhyay and B.B. Pal. "The effect of annealing on the Switching characteristics of an ion-implanted silicon MESFET," IEEE Trans. on Electron Devices, Vol. ED-36, pp.920-929, 1989.

[26] S.N. Chattopadhyay and B.B. Pal, "Analytical modeling an Ion-implanted Silicon MESFET in Post-Anneal condition," IEEE Trans. on Electron Devices, Vol. ED-36, pp.81-87, 1989.

[27] A.H. Khalid and A.A. Rezazadeh, "Fabrication and Characterization of Transparent-Gate Field Effect Transistors Using Indium Tin Oxide," IEEE Proceedings - Optoelectronics, Vol. 143, No. 1, pp. 711, 1996.

[28] T. Sugeta and Y. Mizushima, "High Speed Photoresponse Mechanism of a GaAs MESFET," Japanese Journal of Appl. Physics, Vol. 19, No. 1, pp. L2729, 1980.

[29] K. Kanemoto, et.al, "Dependence of ion implantation: Induced defects on substrate doping," J. Appl.
Phys. Vol.89, pp.3156-3161, 2001.

[30] K.L. Narayanan and M. Yamaguchi, "Phosphorous ion implantation in C60 for the photovoltaic applications," J. Appl. Phys., Vol. 89, pp. 8331-8335, 2001.

[31] H. Seidel, et al. "Anisotropic Etching of Crystalline in Alkaline Solutions," J. Electrochemical Society, Vol. 137, No. 11, pp. 3626-3632, 1990.

[32] M.J. Madou, "Fundamentals of Microfabrication, $2^{\text {nd }}$ Ed.," CRC Press, New York, 2002, p.212.

[33] R. Maboudian, R.T. Howe, "Critical Review: Adhesion in Surface Micromechanical Structures," J. Vac. Sci. Technol. B, vol. 15 (1), p. 1, 1997.

[34] S. Sundararajan, B. Bhushan, "Static Friction and Surface roughness studies of Surface Micromachined Electrostatic Micromotors using an Atomic Force/Friction Force Microscope," J. Vac. Sci. Technol. A, Vol. 19, pp.1777-1785, 2001.

[35] F. Ericson, J. A. Schweitz, "Micromechanical Fracture Strength of Silicon," J. Appl. Phys., Vol. 68, p.5840, 1990 .

[36] T. Yi, C.J. Kim, "Measurement of Mechanical properties for MEMS materials," Meas. Sci. Technol., Vol. 10, pp. 706-716, 1999.

[37] S. Sundararajan, B. Bhushan, et.al, "Mechanical Property Measurements of Nanoscale Structures using an atomic Force Microscope," Ultramicroscopy, Vol. 91, pp. 111-118, 2002.

[38] F. J. Williams, C.M. Aldao, et al, "Why Si (100) Steps are Rougher After Etching," Phy. Rev., B, Condens. Matter, Vol. 55, pp. 13829-13834, 1997.

[39] T. Baum and D.J. Schiffrin, "AFM Study of Surface Finish Improvement by Ultrasound in the Anisotropic Etching of Si (100) in $\mathrm{KOH}$ for Micromaching Applications," J. Micromechanics Microengineering, Vol. 7, pp. 338-342, 1997.

[40] I. Zubel, et al, "Silicon Anisotropic etching in alkaline solutions II On the Influence of anisotropy on the smoothness of etched surfaces," Journal "Sensors and Actuators A: Physical, Vol. 70, pp. 260268, 1998.

[41] M. Shikida, K. Sato, et al, "Differences in anisotropic etching properties of $\mathrm{KOH}$ and TMAH solutions," Journal "Sensors and Actuators A: Physical," Vol. 80, pp.179-188, 2000.

[42] R. Holly and K. Hingerl, "Fabrication of silicon vertical taper structures using $\mathrm{KOH}$ anisotropic 
etching," Microelectronics Engineering, 83, 14301433, 2006.

[43] A. Tabe, A. Uchiyama, et.al in Proceedings of the $1^{\text {st }}$ International Symposium on Semiconductors wafer bonding: Science and Technology, and Applications, U. Gosels, T, Abe, J. Haisma and M.A. Schmidt, Editors, PV 92-7, p 200, The Electrochemical Society Proceedings Series, NJ (1992).

[44] W.P. Maszara, B-L. Jiang et.al, "Role of Surface Morphology in Wafer Bonding," J. Appl. Phys. Vol. 69, p.257, 1991.

[45] H. Angermann and Carola Klimn, "Wet-chemical treatment and electronic interface properties of silicon solar cell," Central European Journal of Physics, Vol. 7, 2, pp.363-370, 2009.

[46] J.D. Hylton, A.R. Burgers and W.C. Sinke, "Alkaline etching of Reflectance reduction in multicrysttaline silicon solar cells," Journal of the Electrochemical Society, Vol. 151, Issue 6, pp.G408-G427, 2004.

[47] S.K. Ghandhi, "VLSI Fabrication Principles - Silicon and Gallium Arsenide, $2^{\text {nd }}$ Ed.," 1994, John Willey \& Sons, Inc.

[48] D.K. Schroder, et al, "Free Carrier Absorption in Silicon," IEEE Trans. On Electron Devices, Vol. ED-25, No. 2, pp. 254-261, 1978.

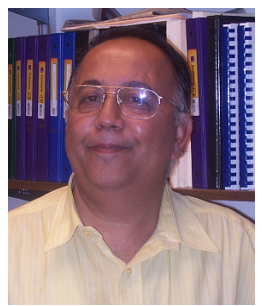

S.N. Chattopadhyay is currently engaged in teaching as a faculty member in the Department Electrical and Computer Engineering, California State University Northridge. He was a faculty of Electrical and Computer Engineering, California State University Fresno from 2002-2004. In 2005, he was awarded a research grant from the US Department of Defense and at present is actively engaged in pursuing an advance research on optoelectronic devices. Recently he guided his team in the successful fabrication of an optically controlled MESFET. He has been awarded the Alexander von Humboldt fellowship in 1993 and pursued research work on indium phosphide epitaxial growth by molecular beam expitaxy and transport characterization in the Technical University of Darmstadt, Germany from 1993-1995. From 1995-1998, he worked as a general manager (head) of the wafer fab of USHA India Limited in collaboration with Samsung. Since 1998-2000, he worked as a team leader of the fabrication group in the Center of Advanced Study in Radio-Physics and Electronics, University of Calcutta, India and successfully developed a SDR IMPATT diode. Currently, he is also actively engaged with research on silicon carbide MESFETs for high power RF amplifier applications.

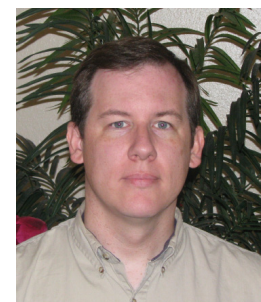

C. B. Overton was born in San Diego, CA, USA in 1970. He worked as a technician at various equipment manufacturers in Santa Barbara, CA, USA from 1989 to 2005. He received his B.S. in Electrical Engineering at California State University, Northridge, USA in 2008. He is currently pursuing his MS degree in Electrical Engineering at California State University, Northridge and is involved with ongoing research on optically controlled MESFET devices and optical Schottky diodes as well as currently working in Burbank, CA as an electronics design engineer in the movie industry.

\section{S. Vetter [not available]}

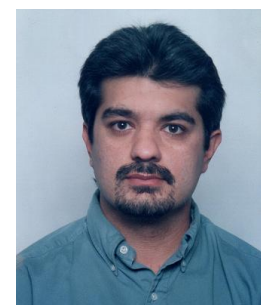

M. Azadeh earned his Bachelor's degree from Tehran University, his master's degree from Portland State University, and his $\mathrm{PhD}$ degree from University of Washington, all in the field of electrical engineering with emphasis in optoelectronics. He has been working as a Senior Design Engineer at Source Photonics Inc. since 2001 specializing in fiber optic transceivers and relevant analog electronics design. $\mathrm{He}$ is a senior member of IEEE. He has published several papers in the areas of electronics, sensors, and optoelectronics, and has authored a book about fiber optics (Fiber Optics Engineering, Springer, 2009). 


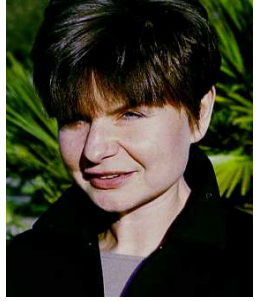

B. H. Olson received Bachelor of Science and Ph.D. degrees in electrical engineering from the University of California at San Diego in 1989 and 1996, respectively. In 1996 she joined the Jet Propulsion Laboratory (JPL) as member of technical staff where she worked on the development of CMOS image sensors. Subsequently she worked for W. L. Gore \& Associates and Micron where she designed parallel channel Fiber Optic Transceivers and commercial CMOS image sensors. In 2003 she became a member of California State Polytechnic University, Pomona's Electrical Engineering faculty.

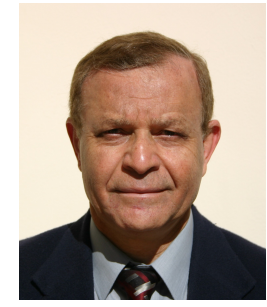

N. El Naga received his Ph.D. degree from the University of Waterloo in 1978. In 1979, he joined the Department of Electrical and Computer Engineering (ECE), California State University Northridge (CSUN) as Assistant Professor and became Professor in 1986. In 2002, he became the Chairman of the Department. Dr. El Naga has received many honors and awards which include the Distinguished Teaching Award, Engineering Council Merit Award, Tau Beta Pi Professor of the year award, College of Engineering and Computer Science Outstanding Faculty award, the university Meritorious Performance and Professionals Promise award, etc. His research interest is focused on Computer Architectures and Digital System design and their applications. He served as a consultant to several companies. He has been awarded several grants and now he is the Principal Investigator of STTR project awarded by the Department of Defense. Dr. El Naga has authored over 37 publications including Technical Reports and papers published and/or presented in internationally reputed scientific and engineering journals and the proceeding of international symposia. He supervised more than 77 graduate theses and projects. 